\title{
A Novel Technique for Copy Move Forgery Detection using Grey Level Co-occurrence Matrix
}

\author{
Parul Sharma, Harpreet Kaur, Simran Uppal
}

\begin{abstract}
In this paper we have studied the GLCM approach as an improvement over SWT-DCT method for feature extraction for CMFD. We have carefully studied the previously used methods and also studied the SWT-DCT method for improvement in features. We have proposed a method for the use of GLCM instead of SWT-DCT method for feature extraction which will improve the results of CMFD method used in the base work framework.
\end{abstract}

Index Terms: CMFD, GLC, SWT.

\section{INTRODUCTION}

The advancement of industrially accessible picture altering apparatuses is the premise of expanding utilization of computerized pictures in our day by day life. Usability of such devices has made control of picture substance simpler. Picture control strategies can be delegated picture steganography and picture falsification. Both picture steganography and picture falsification control a picture yet they vary from each other as indicated by their practices [1]. Steganography controls a subject advanced picture for concealing mystery data while picture fraud changes the first semantic importance of a picture. There have been an extensive number of steganographic systems that use computerized picture for secretive correspondence [4]. In the meantime, different steganalysis systems have been concocted to reveal mystery messages in computerized pictures. The implanted information is reestablished amid the confirmation procedure by contrasting the reference information. From that point, in the measurable examination, the confirmed information is utilized to guarantee the uprightness of advanced media whether fashioned or not. Different existing stenographic devices have likewise been utilized to help criminological specialists for the investigation of interactive media substance. The altering of computerized pictures is extremely simple because of the availability of modern and simple to utilize picture control and altering programming. The altered pictures can be utilized as a beguiling device for concealing the undeniable realities. For example, altered pictures introduced in computerized media misdirect the general population, as imperative articles might be duplicated or hid from the pictures. This displays picture fabrication is a genuine risk; along these lines, it has turned into a key worry for the scientists of advanced picture legal sciences in the ongoing occasions [9]. In writing, we can discover different altering

Revised Manuscript Received on June 15, 2019.

Parul Sharma, Computer science, Chandigarh University, Mohali, India.

Harpreet Kaur, Computer science, Chandigarh University, Mohali, India.

Simran Uppal, Computer science, Chandigarh University, Mohali, India. location procedures that might be sorted as dynamic methods and detached (dazzle) systems.

Duplicate Move is a standout amongst the most widely recognized picture falsification types, where a locale of a picture is reordered into another area of a similar picture [4]. Such a phony is easy to accomplish however difficult to be distinguished as the glued district has similar qualities with the picture. Albeit a lot of calculations have been proposed to handle the duplicate move recognition issue, those calculations contrast in two things; coordinating strategy and kind of highlights. In this paper, we center around dissecting and looking at four coordinating techniques regarding exactness and heartiness against various picture handling tasks. Such investigation and examination give essential data to the structure of new precise and solid duplicate move recognition strategies.

In the present current life, computerized pictures have huge significance since they have turned into a main wellspring of data dispersal. Be that as it may, the accessibility of picture altering instruments made it simpler to manufacture the substance of an advanced picture; making the credibility untruthful. Diverse procedures can be utilized to produce the advanced pictures. Duplicate move phony is the most well known and normal methodology where a particular piece of a picture is reordered somewhere else in a similar picture to cover undesirable part or item. In this investigation [5], they endeavored to review a few inactive square based duplicate move fraud identification procedures. A uninvolved method endeavors to recognize fabrication in advanced pictures with no earlier data. An examination between different methods is additionally included.

\section{Literature SURVEY}

In this paper [6], a novel picture fraud discovery technique is proposed dependent on the steerable pyramid change (SPT) and nearby double example (LBP). In the first place, given a shading picture, we change it in the $\mathrm{YCbCr}$ shading space and apply the SPT change on chrominance channels $\mathrm{Cb}$ and $\mathrm{Cr}$, yielding various multi-scale and multi-arranged subbands. At that point, we depict the surface in each SPT subband utilizing LBP histograms. The histograms from each subband are linked to create a component vector. At long last, a help vector machine utilizes the element vector to group pictures into fashioned or true. The proposed technique has been assessed on three openly accessible picture databases. Our trial results show the viability of the proposed technique and its predominance over some ongoing different strategies.

Duplicate move [7] is a standout amongst the most regularly utilized picture altering activity, where a 
piece of picture content is replicated and after that glued to another piece of a similar picture. So as to make the fraud outwardly persuading and hide its follow, the replicated part may expose to post-preparing tasks, for example, turn and obscure. In this paper, we propose a polar cosine change and estimated closest neighbor looking based duplicate move fabrication identification calculation. The calculation begins by separating the picture into covering patches. Strong and conservative highlights are separated from patches by exploiting the rotationally-invariant and symmetrical properties of the polar cosine change. Potential duplicate move sets are then recognized by distinguishing the patches with comparable highlights, which is detailed as rough closest neighbor looking and achieved by methods for area delicate hashing (LSH). At last, post-confirmations are performed on potential sets to sift through false matches and improve the precision of phony recognition. To whole up, the LSH based comparable fix recognizable proof and the post-check strategies are two noteworthy oddities of the proposed work. Test results uncover that the proposed work can deliver exact recognition results, and it displays high heartiness to different post-handling activities. Also, the LSH based comparative fix discovery plot is significantly more powerful than the generally utilized lexicographical arranging.

As amazing picture [8] altering apparatuses are generally utilized, the interest for recognizing the legitimacy of a picture is tremendously expanded. Duplicate move phony is one of the altering systems which are every now and again utilized. Most existing methods to uncover this fabrication need to improve the heartiness for basic post-handling tasks and neglect to decisively find the altering locale particularly when there are substantial comparative or level areas in the picture. In this paper, a hearty technique dependent on DCT and SVD is proposed to identify this particular antiquity. Right off the bat, the suspicious picture is separated into fixed-measure covering squares and 2D-DCT is connected to each square, at that point the DCT coefficients are quantized by a quantization framework to get a progressively hearty portrayal of each square. Also, each quantized square is separated non-covering sub-squares and SVD is connected to each sub-square, at that point highlights are extricated to lessen the element of each square utilizing its biggest solitary esteem. At long last, the element vectors are lexicographically arranged, and copied picture squares will be coordinated by predefined move recurrence edge. Investigation results exhibit that our proposed strategy can adequately recognize various duplicate move falsification and unequivocally find the copied districts, notwithstanding when a picture was misshaped by Gaussian obscuring, AWGN, JPEG pressure and their blended activities.

The expanding prominence of computerized media and media altering programming [9] has prompted far reaching altering of sight and sound records for malevolent purposes. The most well-known type of altering related with computerized pictures is copy- move phony, in which a bit of a picture is copied and substituted in an alternate area. Accordingly, law implementation and crime scene investigation specialists require dependable and effective methods for recognizing copy- move imitation. This paper proposes a visually impaired crime scene investigation way to deal with the discovery of copy- move falsification. The info picture is divided into covering squares, whereupon a histogram of orientated angles is connected to each square.
Factual highlights are separated and diminished to encourage the estimation of closeness. At long last, highlight vectors are lexicographically arranged, and copied picture squares are recognized by distinguishing comparative square matches after post-handling. Test results exhibit that the proposed strategy can distinguish various instances of copy- move phony and correctly find the copied districts, notwithstanding when managing pictures mutilated by interpretation including little pivots, obscuring, change of brilliance, and shading decrease. We are as of now attempting to improve identification in districts with turn and scaling change over extensive zones.

This work [10] displays another methodology toward duplicate move falsification discovery dependent on multi-scale investigation and casting a ballot procedures of a computerized picture. Given a suspicious picture, we extricate intrigue directs powerful toward scale and pivot discovering conceivable correspondences among them. We bunch reporter focuses into districts dependent on geometric requirements. From that point, we develop a multi-scale picture portrayal and for each scale, we inspect the produced gatherings utilizing a descriptor emphatically strong to pivot, scaling and somewhat hearty to pressure, which diminishes the inquiry space of copied districts and yields a discovery map. A ultimate choice depends on a casting a ballot procedure among all recognition maps. We approve the strategy utilizing different datasets including unique and reasonable picture cloning. We contrast the proposed technique with 15 others from the writing and report promising outcomes.

Area duplication [11] imitation is one of most normal altering guiles. A few strategies have been created to identify and find the altered locale, while most techniques do come up short when the duplicated district is pivoted before being stuck on account of the de-synchronization in the seeking system. To take care of the issue, the paper proposes a productive and strong latent validation technique that utilizes the circle square and the $\mathrm{Hu}$ minutes to recognize and find the copy locales with pivot. Test results demonstrate that our strategy is strong not exclusively to clamor pollution, obscuring and JPEG pressure, yet in addition to the turn. In the interim, the proposed technique has better time execution contrasted and leaving strategies in light of the lower highlight measurement.

As new advances [12] and gadgets are presented in the market, the wrongdoing rate likewise increments in creating and created nations. One such wrongdoing is picture imitation which can be distinguished by legal applications. In this paper, they propose an original thought for recognizing fabrication assault done by haze ancient rarity not at all like existing fraud assault done by geometrical mutilation, for example, pivot and scaling. The proposed strategy section locale of enthusiasm from the info phony picture dependent on the blend of factual investigation with shading surface examination which incorporates obscure curio district. For every district of intrigue, we propose another strategy for evaluating level of haze to isolate fashioned haze antiquity and typical haze curio. So as to approve the recognized produced obscure antiquity, we investigate Fourier and Gabor surface highlights to think about the structure of the manufactured haze curio which kills false haze fashioned haze ancient rarity. To assess the

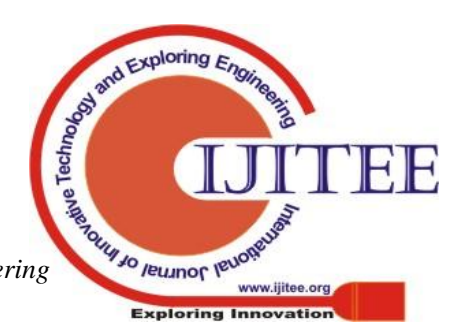


proposed fashioned obscured locale identification technique, we utilize two standard databases specifically, Image information control, and MICC-F220 for experimentation. Trial consequences of the proposed strategy with existing techniques demonstrate that the proposed strategy outflanks the current strategies regarding produced obscure relic locale recognition.

Highlight Extraction [13] is a strategy for catching visual substance of pictures for ordering and recovery. Crude or low dimension picture highlights can be either broad highlights, for example, extraction of shading, surface and shape or space explicit highlights. This paper introduces a use of dim dimension co-event grid (GLCM) to extricate second request measurable surface highlights for movement estimation of pictures. The Four highlights specifically, Angular Second Moment, Correlation, Inverse Difference Moment, and Entropy are registered utilizing Xilinx FPGA. The outcomes demonstrate that these surface highlights have high separation precision, requires less calculation time and henceforth productively utilized for continuous Pattern acknowledgment applications.

\section{CMFD USING SWT-DCT}

In the recently proposed method a key commitment [13] is proposed dependent on SWT and DCT for CMF discovery and restriction. The purpose behind choosing the SWT is its interpretation invariance and restriction properties in both phantom and spatial areas. The proposed system disintegrates the information picture being referred to into four sub groups (estimation, flat, vertical and slanting) through the interpretation invariant SWT. The procedure further partitions the estimation sub band into covering hinders for highlight extraction. A decreased element of the component is gotten by applying DCT to each covering square. The inspiration to utilize DCT over the covering squares of estimate sub band of SWT is its vigor for post-handling tasks, for example scaling, pressure, and obscuring, as these activities are usually connected to the altered pictures to make the altering location troublesome. Along these lines, the blend of SWT and DCT makes the portrayal of highlights increasingly various and furthermore shows up as a superior decision for CMFD.

\section{CMFD USING GLCM}

Use Dark dimension simultaneousness grid [14] will be utilized for the element extraction in the as of now recently proposed technique rather than SWT-DCT. A GLCM is where the quantity of lines and sections is equivalent to the quantity of dim dimensions, $\mathrm{G}$, in the picture. The framework component $P(I, j \mid \Delta x, \Delta y)$ is the relative recurrence with which two pixels, isolated by a pixel remove $(\Delta x, \Delta y)$, happen inside a given neighborhood, one with force 'I' and the other with power ' $j$ '. The lattice component $P(I, j \mid d, \theta)$ contains the second request likelihood esteems for changes between dark dimensions 'I' and 'j' at a specific uprooting separation $d$ and at a specific edge $(\Theta)$. Utilizing countless dimensions $G$ infers putting away a great deal of brief information, for example a $\mathrm{G} \times \mathrm{G}$ grid for every mix of $(\Delta x$, $\Delta y)$ or $(\mathrm{d}, \Theta)$. Because of their expansive dimensionality, the GLCM's are delicate to the extent of the surface examples on which they are evaluated. Accordingly, the quantity of dark dimensions is regularly decreased. GLCM lattice detailing can be clarified with the case for four diverse dim dimensions. Here one pixel counterbalance is utilized (a reference pixel and its prompt neighbor). In the event that the window is sufficiently vast, utilizing a bigger counterbalance is conceivable. The upper left cell will be loaded up with the occasions the mix 0,0 happens, for example what number of time inside the picture region a pixel with dark dimension 0 (neighbor pixel) tumbles to one side of another pixel with dim dimension 0 (reference pixel). For better component extraction we are utilizing GLCM.

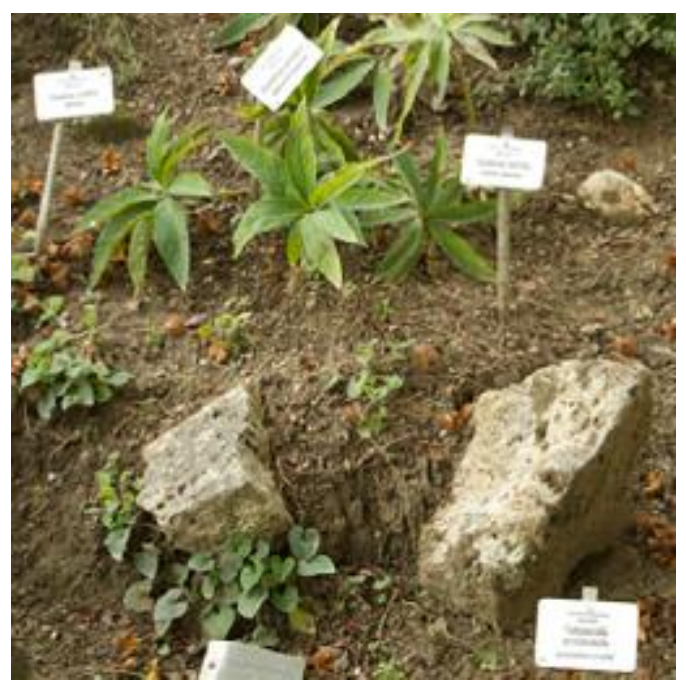

Fig 1(a): Original Image

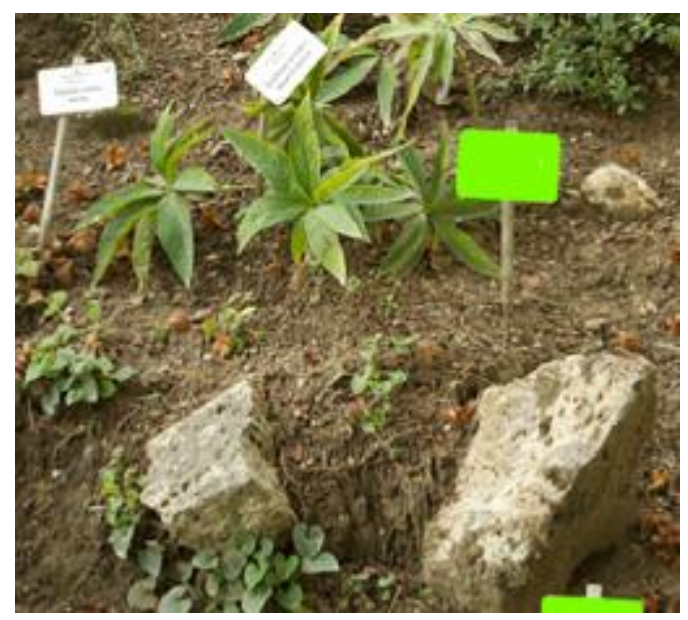

Fig 1(b): Forgery detected 


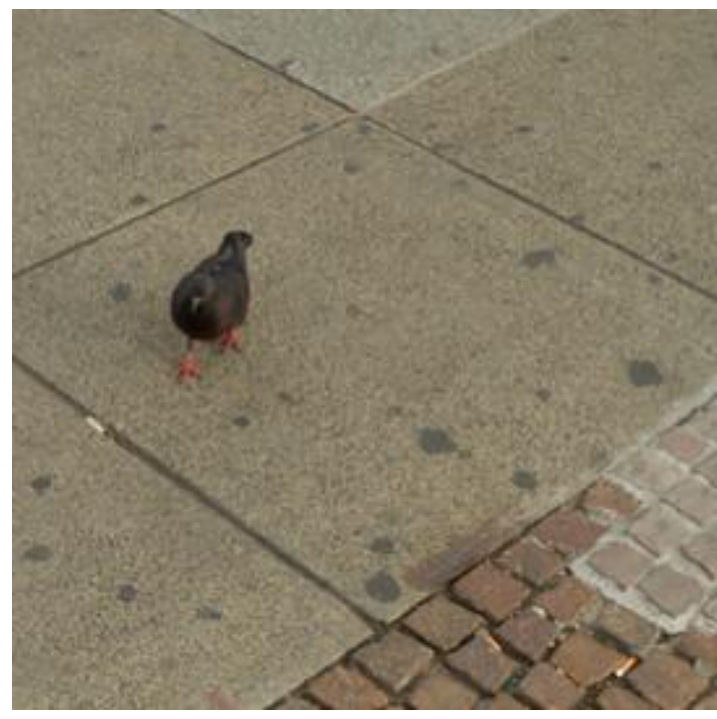

Fig 2(a): Original Image

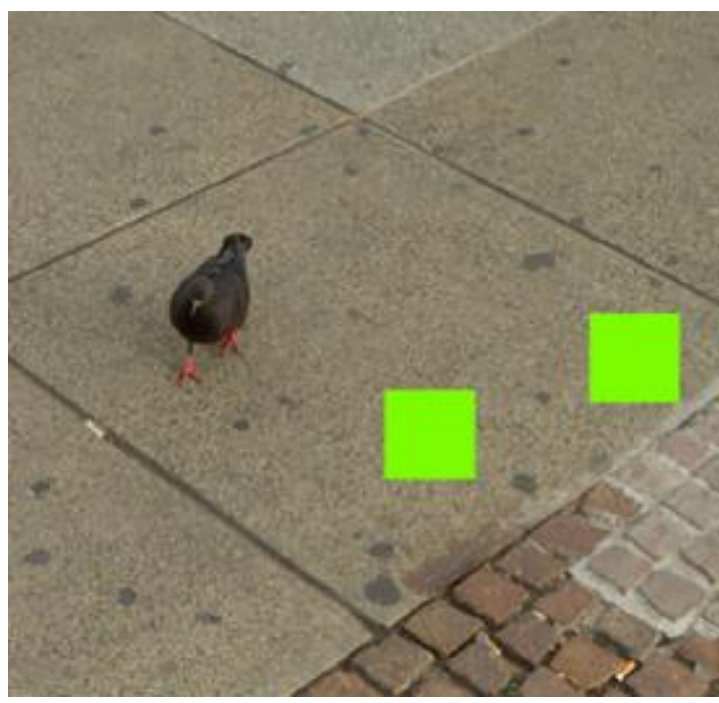

Fig 2(b): Forgery detected

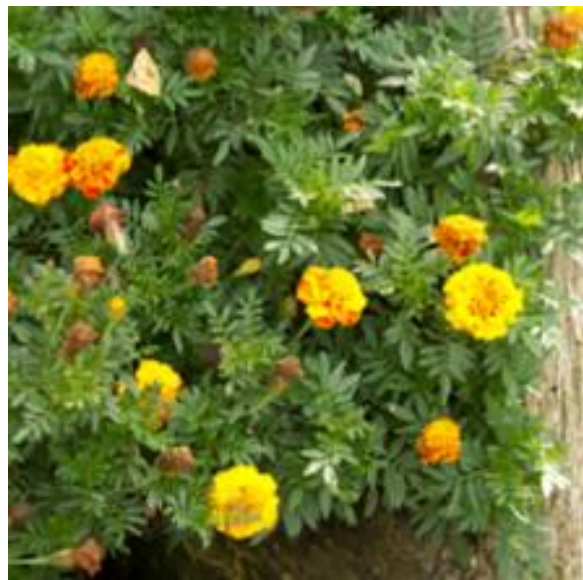

Fig 3(a): Original Image

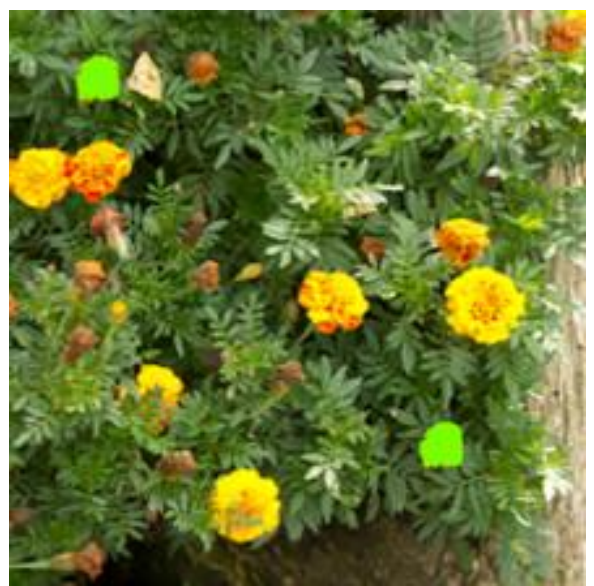

Fig3(b): Forgery detected

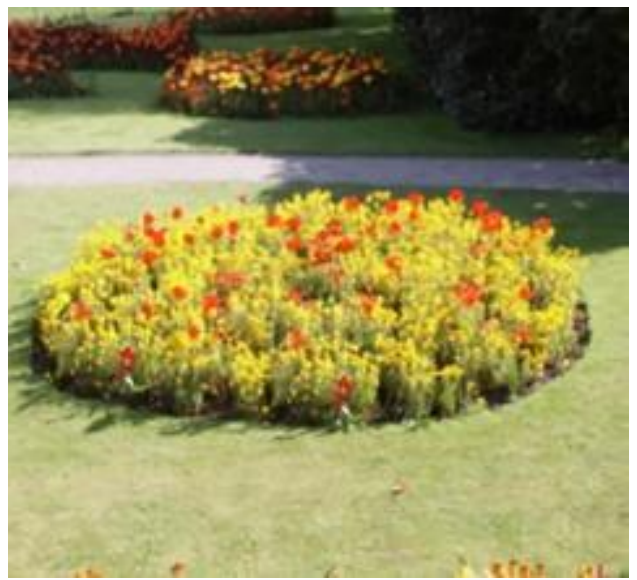

Fig 4(a): Original Image

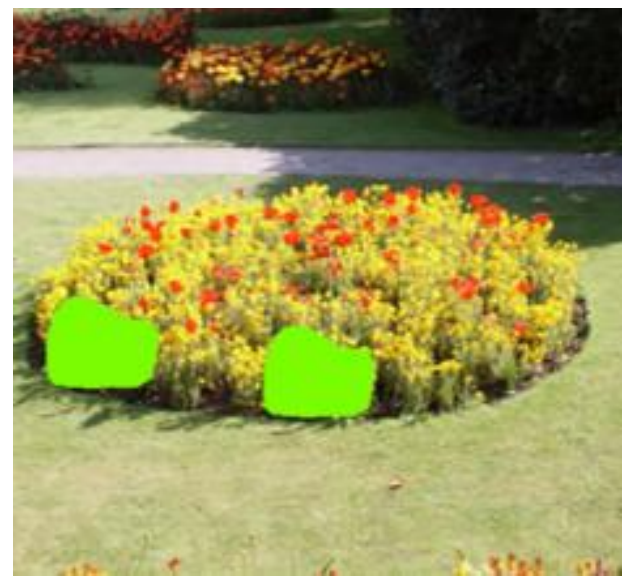

Fig 4(b): Forgery detected

\section{OTHER REFINED METHODS}

In our search for different CMFD methods we found some other techniques like Sorted neighborhood [15] method and DWT-PCA (EVD) [16] bases methods. Other methods like DWT- HU based CMFD [17], Transient invariant de-noising [18], Wavelet decomposition [19], Passive method using wavelet transform [20], Forensic technique exposing region decomposition [21], Robust detection algorithm [22] and localization of double compression. Other surveys

\section{Published By:}

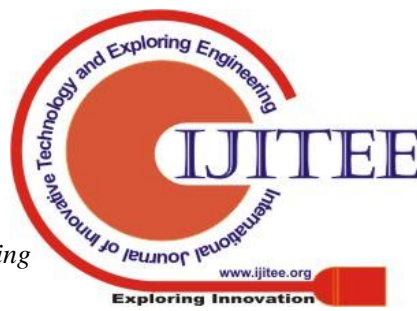


were also found with similar methods. One more forensic method based on stationary wavelets [25] was also found unlike the previous one.

\section{COMPARISON}

After the care discourse of the topic we have found following predictive points of improvements in the previously proposed method.

TABLE I: CMFD comparison of SWT-DCT and GLCM

\begin{tabular}{|c|c|c|}
\hline Parameter & CMFD with DCT-SWT & CMFD with GLCM \\
\hline Average detection rate & Moderate & Low \\
\hline BME & Moderate & Very High \\
\hline SNR & High & Expected to decrease \\
\hline Entropy & Moderate & Expected to decrease \\
\hline Homogenity & High & \\
\hline
\end{tabular}

7. Li, Y. (2013). Image copy-move forgery detection based on polar cosine transform and approximate nearest neighbor searching. Forensic science international, 224(1-3), 59-67.

\section{CONCLUSION}

This research tries to highlight the advancement the GLCM is going to have if we replace SWT-DCT method in the existing framework for CMFD. We have given detailed explanation of SWT-DCT based method for CMFD and how it works in different parts and then analyzed the used of GLCM. In our analysis GLCM based CMFD model is expected to perform better than the SWT-DCT based CMFD in Average detection rate, BME, SNR, Entropy and Homogeneity.

\section{REFERENCES}

1. Islam, M., Shah, M., Khan, Z., Mahmood, T., \& Khan, M. J. (2015, December). A new symmetric key encryption algorithm using images as secret keys. In 2015 13th International Conference on Frontiers of Information Technology (FIT) (pp. 1-5). IEEE.

2. Kessler, G. C. (2004). An overview of steganography for the computer forensics examiner. Forensic science communications, 6(3), 1-27.

3. Almohammad, A., Hierons, R. M., \& Ghinea, G. (2008, March). High capacity steganographic method based upon JPEG. In 2008 Third International Conference on Availability, Reliability and Security (pp. 544-549). IEEE.

4. Al-Qershi, O. M., \& Khoo, B. E. (2017). Comparison of matching methods for copy-move image forgery detection. In 9th International Conference on Robotic, Vision, Signal Processing and Power Applications (pp. 209-218). Springer, Singapore.

5. Mahmood, T., Nawaz, T., Ashraf, R., Shah, M., Khan, Z., Irtaza, A., \& Mehmood, Z. (2015, December). A survey on block based copy move image forgery detection techniques. In 2015 International Conference on Emerging Technologies (ICET) (pp. 1-6). IEEE.

6. Muhammad, G., Al-Hammadi, M. H., Hussain, M., \& Bebis, G. (2014). Image forgery detection using steerable pyramid transform and local binary pattern. Machine Vision and Applications, 25(4), 985-995.
8. Zhao, J., \& Guo, J. (2013). Passive forensics for copy-move image forgery using a method based on DCT and SVD. Forensic science international, 233(1-3), 158-166.

9. Lee, J. C., Chang, C. P., \& Chen, W. K. (2015). Detection of copy-move image forgery using histogram of orientated gradients. Information Sciences, 321, 250-262.

10. Silva, E., Carvalho, T., Ferreira, A., \& Rocha, A. (2015). Going deeper into copy-move forgery detection: Exploring image telltales via multi-scale analysis and voting processes. Journal of Visual Communication and Image Representation, 29, 16-32.

11. Liu, G., Wang, J., Lian, S., \& Wang, Z. (2011). A passive image authentication scheme for detecting region-duplication forgery with rotation. Journal of Network and Computer Applications, 34(5), 1557-1565.

12. Uliyan, D. M., Jalab, H. A., Wahab, A. W. A., Shivakumara, P., \& Sadeghi, S. (2016). A novel forged blurred region detection system for image forensic applications. Expert Systems with Applications, 64, 1-10.

13. Mohanaiah, P., Sathyanarayana, P., \& GuruKumar, L. (2013). Image texture feature extraction using GLCM approach. International journal of scientific and research publications, $3(5), 1$.

14. Lee, J. C. (2015). Copy-move image forgery detection based on Gabor magnitude. Journal of Visual Communication and Image Representation, 31, 320-334.

15. Li, G., Wu, Q., Tu, D., \& Sun, S. (2007, July). A sorted neighborhood approach for detecting duplicated regions in image forgeries based on DWT and SVD. In 2007 IEEE international conference on multimedia and expo (pp. 1750-1753). IEEE.

16. Zimba, M., \& Xingming, S. (2011). DWT-PCA(EVD) Based Copy-move Image Forgery Detection. International Journal of Digital Content Technology and its Applications, 5(1).

17. Mahmood, T., Nawaz, T., Shah, M., Khan, Z., Ashraf, R., \& Habib, H. A. (2016). Copy-move forgery detection technique based on DWT and $\mathrm{Hu}$ Moments. International Journal of Computer Science and Information Security (IJCSIS), 14(5).

18. Coifman, R. R., \& Donoho, D. L. (1995). Translation-invariant de-noising. In Wavelets and statistics (pp. 125-150). Springer, New York, NY.

19. Starck, J. L., Fadili, J., \& Murtagh, F. (2007). The undecimated wavelet decomposition and its reconstruction. IEEE Transactions on Image Processing, 16(2), 297-309.

20. Muhammad 
G. (2012). Passive copy move image forgery detection using undecimated dyadic wavelet transform. Digital investigation, $9(1), 49-57$.

21. Mahmood, T., Mehmood, Z., Shah, M., \& Khan, Z. (2018). An efficient forensic technique for exposing region duplication forgery in digital images. Applied Intelligence, 48(7), 1791-1801.

22. Cao, Y., Gao, T., Fan, L., \& Yang, Q. (2012). A robust detection algorithm for copy-move forgery in digital images. Forensic science international, 214(1-3), 33-43.

23. Amerini, I., Uricchio, T., Ballan, L., \& Caldelli, R. (2017, July). Localization of JPEG double compression through multi-domain convolutional neural networks. In 2017 IEEE Conference on computer vision and pattern recognition workshops (CVPRW) (pp. 1865-1871). IEEE.

24. Chaplot, S., Patnaik, L. M., \& Jagannathan, N. R. (2006). Classification of magnetic resonance brain images using wavelets as input to support vector machine and neural network. Biomedical signal processing and control, 1(1), 86-92.

25. Mahmood, T., Nawaz, T., Mehmood, Z., Khan, Z., Shah, M., \& Ashraf, R. (2016, August). Forensic analysis of copy-move forgery in digital images using the stationary wavelets. In 2016 Sixth International Conference on Innovative Computing Technology (INTECH) (pp. 578-583). IEEE.

\section{AUTHORS PROFILE}

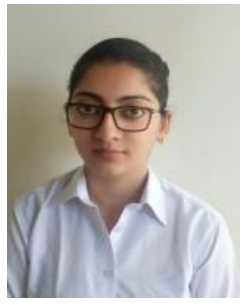

Parul Sharma: Qualification: B.E in electronics and communication engineering from Bahra University, Shimla, in 2017 with specialization in web application. Pursuing Master of engineering in Electronics and communication from Chandigarh University, Punjab, with the specialization in image processing and recently worked on forensic based application in image processing, which works in finding out various kind of manipulation in an image.

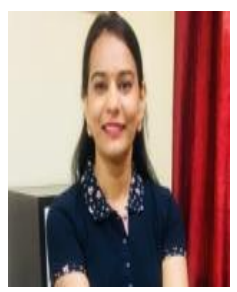

Harpreet Kaur: Assistant professor in Chandigarh university, eight students guided in M.E thesis. Published number of articles in reputed journals, national as well as international conferences. Areas of specialization are optimization, image processing and database

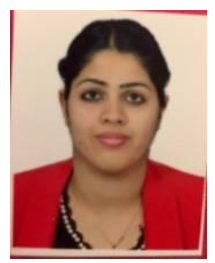

Simran Uppal: Qualification: B.E in electronics and communication engineering from RIMT institute of engineering, Gobindgarh, with specialization in networking. Pursuing masters in electronics and communication engineering from Chandigarh University with specialization in image processing and worked in the steganography based application. 